\title{
Los dos modelos de enlace entre la teoría y la práctica según la Introducción a la Crítica de la facultad de juzgar de Immanuel Kant
}

\author{
NATALIA ANDREA LERUSSI \\ Universidad de Buenos Aires - CONICET (Argentina) \\ natalialerussi@gmail.com
}

\begin{abstract}
Resumen
En el trabajo nos ocupamos de desarrollar el problema del enlace entre la filosofía teórica y la filosofía práctica o del tránsito entre naturaleza y libertad tal y como Kant lo aborda en la Introducción definitiva a la Crítica de la facultad de juzgar. Específicamente proponemos la hipótesis según la cual Kant describe el modo como dicho enlace se realiza, a través de la facultad de juzgar, a partir de dos modelos diferentes. Según el primer modelo, el tránsito se efectúa a partir de la idea de un fundamento de unidad de lo suprasensible que subyace a la naturaleza y lo suprasensible por libertad. De acuerdo al segundo modelo, el tránsito es posible porque lo suprasensible por libertad es puesto como causa o fundamento de la ley natural. Nos enfrentamos, así, a dos modos de pensar diferentes el tránsito en cuestión y, por lo tanto, dos modos altemativos de pensar la realización de la libertad en la naturaleza.
\end{abstract}

Palabras claves: Enlace, teoría vs. práctica, facultad de juzgar, suprasensible.

\section{The two Models of linking Theory and Practice according to the Introduction to the Critique of the Power of Judgment of Immanuel Kant}

\begin{abstract}
In the paper I develop the question of linking theory and practice or, in other words, the problem of the transition between nature and freedom in Kant's (definitive) Introduction to his Critique of the Power of Judgment. I propose specifically the bypotheses according to which Kant describes indeed two different models through which the linking occurs. According to the first one the transition accomplishes thanks to the idea of a ground of the unite of the supersensible that would underlie nature and the supersensible presupposed by freedom. According to the second one, the transition is possible because the supersensible by freedom is put it as cause or ground of the law of nature. Thus we face to two different ways of thinking the linking between theory and practice and, consequently, two alternative ways of thinking the realization of freedom in nature.
\end{abstract}

Key words: Linking, theory vs. practice, judgement, supersensible.

Doctora en Filosofía por la Universidad Nacional de Córdoba (Argentina), becaria posdoctoral del Conicet y docente en la cátedra de Historia de la Filosofía Moderna en la Universidad de Buenos Aires. Algunas de sus contribuciones recientes son: "Acerca de la analogía de la razón con lo orgánico. Reflexiones en torno a la expresión epigénesis de la razón pura en Kritik der reinen Vernunft B \$27" (2014) y “La teoría kantiana de las razas y el origen de la epigénesis” (2013). 


\section{Introducción}

La tendencia de las interpretaciones de la Crítica de la facultad de juzgar (CFJ) en las últimas décadas del siglo XIX y comienzos del siglo XX fue leer la obra de forma no unitaria o sistemática. Así, se interpretó la obra o bien como una «Crítica del gusto» (Cohen, 1889) ${ }^{1}$, o bien, a partir de la Segunda parte (la «Crítica de la facultad de juzgar teleológica»), como una novedosa teoría vitalista de la naturaleza (Ungerer, 1922; Driesch, 1924). En un caso o en otro, la composición entera y unitaria de la obra no era tenida en cuenta.

A finales de los 60's Düsing (1968: 102) defiende la unidad de la obra a partir de lo que él considera su «objetivo principal», esto es, el enlace entre la filosofía teórica y la filosofía práctica. Unos años después de esto Bartuschat (1972: 8) señala que gracias al abordaje del problema subrayado por Düsing, la CFJ alcanza un lugar sistemático en la obra kantiana. El problema del enlace entre la teoría y la práctica o, en los términos con los que también Kant enuncia este problema, la cuestión del tránsito entre naturaleza y libertad o de la realización de la libertad en la naturaleza, se transforma desde entonces en un problema fundamental de la $\mathrm{CFJ}^{2}$.

Aunque desde entonces hay un acuerdo entre los críticos en que es a través de la facultad de juzgar que se produce dicho tránsito no hay consentimiento sobre cómo esto ocurre, efectivamente, según la $\mathrm{CFJ}^{3}$. Por un lado, hay un grupo de intérpretes que sostienen que a través de la facultad de juzgar teleológica (tal y como se desarrolla en $\$ 83$ de la «Metodología a la Crítica de la facultad de juzgar teleológica») se establece el

1 En general, la corriente neokantiana, no atendió a la importancia que tiene la Segunda Parte de la obra, la «Crítica de la facultad de juzgar teleológica», en la concepción kantiana de naturaleza. Para este grupo de intérpretes (Stadler, 1874; Vaihinger, 1913 [1911]; Bauch, 1917: 446; Cassirer, 1994 [1918]; Hartmann, 1926: 41) la teleología kantiana es sólo una metodología de la investigación de la naturaleza de los organismos (la cual podría ser reducida al mecanismo natural).

2 Según nuestros registros, entre la crítica contemporánea, el primer texto dedicado al problema específico del tránsito entre las «dos partes de la filosofía» según Kant es la tesis doctoral de Max Horkheimer defendida en 1925. En dicha obra el autor señala (Horkheimer, 1925: 64), sin embargo, que la facultad de juzgar no soluciona el problema del «abismo» entre las dos partes de la filosofía puesto que «los reinos de la razón teórica y práctica» se reducen, en su interpretación, a lo teórico.

3 En adelante se cita la obra de Kant según las siguientes traducciones: Kant, 1991, 2001, 2004, 2007a y 2007b. La ubicación de la referncia se indica conforme a la edición de la Akademie Ausgabe, en adelante, AA (Kant, 1902ss), exceptuando la Crítica de la razón pura (CRP) que se cita, según el modo habitual, CRP A (edición 1781) y/o B (edición 1787. 
tránsito entre naturaleza y libertad. Esto significa que es mediante la cultura o la historia de la cultura que se realizarían en la naturaleza los fines de la libertad. En los términos de Yovel (1989: 31) «la historia es entonces el proceso de la sintesis requerida entre la libertad y la naturaleza» (esta línea de lectura ha sido defendida por varios intérpretes: Düsing, 1968: 103, 105, 216; Bartuschat, 1972: 245; Anderson-Gold, 1981: 3; Turro, 1996; Wood, 1999: 309; Allison, 2001: 208/218, 2009: 41, entre otros). Por otro lado, otro grupo de intérpretes defiende que es a través del arte y/o del gusto que se efectúa el tránsito en cuestión. Así, según Mertens (1975: 234), el indicador del tránsito entre naturaleza y libertad es la belleza de la naturaleza y el arte, asimismo Michael Rohlf (2008: 344) señala que «el tránsito es posible sólo a través de un principio a priori para nuestra facultad del gusto» (véase también: Schrader, 1953/1954: 205, 218). Los intérpretes no han advertido, sin embargo, que los términos con los cuales Kant enuncia la cuestión del enlace entre la teoría y la práctica a través de la facultad de juzgar (antes de su especificación en facultad de juzgar estética y facultad de juzgar teleológica) no son unívocos. Por eso, a continuación dejaremos entre paréntesis la disputa sobre si es la teleología o el gusto aquello que habilita el tránsito y abordaremos el problema más primitivo de definir los marcos conceptuales mediante los que Kant describe la cuestión, en sentido general, en la Introducción definiti$\mathrm{va}^{4}$ a la obra. Según la hipótesis que defendemos Kant presenta en esta Introducción, en realidad, dos modelos diferentes de efectuación del tránsito a través de la facultad de juzgar, el primero, en el apartado II, el segundo, en el apartado IX y último de esta Introducción. Para los dos modelos, el concepto de lo suprasensible es fundamental aunque, para el primer modelo, el tránsito se realiza a partir de la suposición de un fundamento de unidad de lo suprasensible que subyace a la naturaleza y lo suprasensible por libertad. De acuerdo al segundo modelo, el tránsito es

$4 \quad$ Kant escribió dos Introducciones a la CFJ, esto es, además de la «Introducción definitiva», una extensa primera introducción a la obra a la que referimos, según el uso habitual, como «Primera Introducción». Kant señaló a su editor que desechaba esta introducción a causa de su larga extensión (véase: AA, XI, 143). Dicho manuscrito fue enviado por Kant en 1792 a su amigo Jacob S. Beck quien en 1797 lo publicó en forma mutilada. En este formato fue publicado el texto varias veces durante el siglo XIX. Recién en 1914 el manuscrito fue publicado íntegramente por Otto Bueck en el quinto tomo de los Immanuel Kants Werke editado por Ernst Cassirer (véase: Cassirer, 1970 [1938]: 97). Aunque el argumento expreso de Kant para desechar este texto como Introducción a la CFJ no fue su contenido sino su extensión, así, Cassirer (1970: 97) señala que «no h[a] sido capaz de descubrir ninguna diferencia importante entre las dos introducciones»), el cuidadoso estudio filológico de ambos textos condujo a Tonelli (1954: 444) a afirmar que la Introducción definitiva «más que acortar» el contenido de la Primera, «la reemplaza». 
posible porque lo suprasensible por libertad es puesto como causa o fundamento de la ley natural. Se trata así de dos modos de pensar diferentes el tránsito en cuestión, esto es, dos maneras alternativas de pensar la realización de la libertad en la naturaleza.

Tomando en consideración la Introducción definitiva a la CFJ, en el primer apartado (1) presentamos la tesis de la necesaria separación entre la teoría y la práctica según Kant así como también la cuestión de por qué esto se presentaría como un problema para el mismo filósofo; en el segundo apartado (2) mostramos que Kant entiende la facultad de juzgar y su principio, la conformidad a fin, como el medio del enlace entre la teoría y la práctica; en el último apartado (3) defenderemos nuestra hipótesis según la cual Kant presentaría en esta Introducción dos modelos alternativos para pensar el modo como la facultad de juzgar piensa el tránsito entre naturaleza y libertad.

\section{Planteamiento del problema del enlace entre la filosofía teórica y práctica: dos legislaciones sobre el mismo suelo de la experiencia}

Además del objetivo de poner al descubierto un principio para la facultad de juzgar, la CFJ tiene una finalidad sistemática cuya importancia no es menor que el primero. Se trata de enfrentar el problema del enlace entre las dos partes de la filosofía. De hecho, el apartado III de la Introducción definitiva lleva expresamente por título «De la crítica de la facultad de juzgar como un medio de enlace (Übergang) entre las dos partes de la filosofía en un todo».

La cuestión de un medio de enlace entre la filosofía teórica y práctica o, en los términos con los que Kant a veces pone esta cuestión, el problema del tránsito entre los dos dominios de la razón, la naturaleza y la libertad, es mencionada por Kant antes de la CFJ en algunas ocasiones. ${ }^{5}$ Por ejemplo, en la «Dialéctica trascendental» a la Crítica de la Razón Pura (CRP) Kant sostiene (Kant, 2007a; CRP, A329/ B385) que los conceptos de la razón (esto es, las ideas trascendentales) «quizás» efectúen un «trán-

\footnotetext{
$5 \quad$ Según el recuento de Allison (2001: 197) Kant hace mención de este problema antes de la CFJ en cuatro ocasiones: CRP (Kant, 2007a; CRP, A329/B386); en la Crítica de la Razón Práctica (Kant, 2007b; AA, V, 7); implícitamente en Sobre el uso de principios teleológicos en filosofía (Kant, 2004; AA, VIII, 182-183) y en la sección XI de la Primera Introducción a la CFJ (Kant, 1991; AA, XX, 55ss). Düsing (1968: 50, 102) sostiene que "por primera vez en la Crítica de la facultad de juagar y, fundamentalmente en las dos Introducciones, Kant investiga más de cerca la particularidad del concepto de conformidad a fin en relación con la naturaleza y la libertad». Sin embargo, según este intérprete, el tema alcanza un lugar sistemático «sólo en la segunda Introducción», es decir, en la llamada por nosotros «Introducción definitiva».
} 
sito de los conceptos de la naturaleza a los prácticos» de manera tal que las mismas «puedan darles a las ideas morales mismas apoyo y concatenación con los conocimientos especulativos de la razón». Kant señala a continuación que sobre esta cuestión «debe esperarse» «la explicación», sin embargo, dicha explicación no es ofrecida en esta obra. Más aún, en la Crítica de la Razón Práctica (CRPr) (Kant, 2007b; AA, V, 91) Kant supone que la efectuación de dicho tránsito no ha sido alcanzada aún, por el contrario, presenta la esperanza de que «acaso llegue un día en que lleven [ciertas comparaciones] a comprender la unidad de toda la facultad de la razón pura (de la teórica como de la práctica) y pueda deducirse todo de un principio». Se trata de un problema que es «inevitable» para la razón humana "que sólo encuentra cabal satisfacción en una unidad completamente sistemática de sus conocimientos».

Ahora, ¿en qué sentido la filosofía teórica y la filosofía práctica y sus objetos, naturaleza y libertad, se encuentran separados? Y luego, ¿por qué esta brecha se presenta como un problema? En la sección II de la Introducción definitiva a la CFJ titulado «Del dominio de la filosofía en general» Kant (1991; AA, V, 174/5) presenta una serie de conceptos que expresan la necesidad de separar los conceptos de naturaleza y libertad y, al mismo tiempo, el problema de pensar, sin embargo, un tránsito entre los mismos. Dichos conceptos son los de dominio (Gebiet, ditio) y suelo (Boden, territorium) y campo (Feld).

Según Kant, los conceptos en tanto referidos a objetos pueden tener un campo, un suelo y/o un dominio. La distinción entre los mismos depende de la relación que mantiene el objeto de aquellos conceptos con nuestras facultades de conocimiento. Así, el campo es el conjunto de conceptos que no contienen contradicción lógica interna, esto es, que son lógicamente posibles o pensables, independientemente de si el objeto al que aquellos corresponden puede o no ser conocido por nosotros. Pertenecen al campo de los conceptos todos los conceptos no contradictorios como, por ejemplo, los de sustancia, árbol, alma y minotauro. Ahora, en el interior de dicho campo hay una región en la cual el conocimiento es posible, esto es, cuyos objetos pueden ser experimentados directa o indirectamente por nosotros. A esta región Kant la llama el suelo de dichos conceptos. Así, todos los conceptos empíricos, como por ejemplo, árbol o flogisto pertenecen al suelo de los conceptos pues sus objetos pueden ser conocidos por nosotros, directa o indirectamente, a través de la experiencia. Ahora bien, dentro del área de los conceptos cuyos objetos podemos conocer se distingue un conjunto que, aun cuando no son extraídos de la experiencia, se refieren a y ejercen un cierto derecho sobre la misma. Dichos conceptos definen un dominio y se corresponden con los conceptos a priori en tanto son referidos a objetos. Según Kant, se pue- 
den diferenciar dos y sólo dos tipos de conceptos a priori en tanto referidos a objetos, estos es, exclusivamente dos dominios. Kant (1991; AA, $\mathrm{V}, 174)$ señala:

Nuestra entera facultad de conocimiento tiene dos dominios, el de los conceptos de la naturaleza y el del concepto de la libertad, pues por medio de ambos es legislativa a priori.

Se trata de dos legislaciones que representan objetos separados por un «abismo inabarcable», lo sensible o fenoménico, por un lado y la cosa en sí o lo suprasensible, por otro. Hacia el final de la Introducción definitiva, Kant (1991; AA, V, 195) afirma:

El dominio del concepto de la naturaleza bajo la primera legislación [la legislación del entendimiento], y el del concepto de libertad bajo la otra [la legislación de la razón], están completamente segregados (...) por el gran abismo que separa lo suprasensible de los fenómenos.

Esta segregación entre ambos dominios implica, muy especialmente, que ninguno puede ser reducido ni al otro ni a un principio superior. En los términos del filósofo (Kant, 1991; AA, V, 175):

Que estos dos dominios (...) no constituyan uno solo, proviene de que el concepto de la naturaleza torna a sus objetos aptos para la representación, en la intuición, aunque no como cosas en sí mismas, sino como mero fenómenos, y el concepto de la libertad, al contrario, torna en su objeto apta para la representación a una cosa en sí, aunque no en la intuición, y que, por tanto, ninguno de los dos puede proporcionar un conocimiento teórico de su objeto (...) como cosa en sí, que sería lo suprasensible.

Puesto que las dimensiones en las que se representan su objeto ambas legislaciones son diferentes, no se produce un conflicto de competencia entre los dominios implicados y, por lo tanto, tampoco se produce la necesidad de resolver un conflicto entre las mismas bajo las estrategias de reunirlas bajo reducción de o bien una a la otra o bien de ambas a otra superior. En el dominio de la naturaleza se encuentran los conceptos y principios a los que debe ajustarse la naturaleza a fin de que el conocimiento de la misma sea posible, legisla este dominio sobre los objetos en tanto se presentan a nuestra intuición y nada nos informa sobre cómo ha de ser la naturaleza independientemente del modo como la conocemos. El domino de la libertad, por otro lado, en tanto legislación a priori, determina el principio al que debe ajustarse una voluntad libre. El concepto de voluntad libre no es tal cuyo objeto nos sea dado en la experiencia; por 
el contrario, es la idea de un agente que actúa independientemente de los principios de la experiencia y en conformidad a una ley racional (moral). La idea de una voluntad libre apunta, consiguientemente, a un más allá de la experiencia, es decir, a la cosa en sí. El dominio de la libertad determina a una voluntad como cosa en sí desde un punto de vista práctico, determinando la condición a la que debemos ajustar nuestras máximas de acción para que ésta sea racional (moral). Estas tesis son uno de los núcleos centrales de la filosofía kantiana: a fin de garantizar el conocimiento científico, por un lado, el dominio de la naturaleza, la ley natural, debe ejercer su legislación en toda la naturaleza, incluso en la naturaleza humana, a fin de garantizar la libertad, por otro lado, nuestra voluntad debe pensarse como agente que actúa por fuera del ámbito de la naturaleza. Kant (1991; AA, V, 175) señala en este contexto que la posibilidad de pensar «al menos sin contradicción la coexistencia de ambas legislaciones (...) fue demostrada por la Crítica de la razón pura, al aniquilar las objeciones en su contra a través del descubrimiento de la ilusión dialéctica». La resolución de la contradicción (aparente) entre la ley de causalidad según las leyes de la naturaleza, principio de conocimiento de las cosas en la intuición, y la causalidad como libertad, principio de determinación de una voluntad libre, es desarrollada, de hecho, en la representación del tercer conflicto de la antinomia de la razón (Kant, 2007a; CRP, A444/ B472ss).

Ahora, la necesidad de separar ambas legislaciones, el énfasis en que no es posible establecer un puente entre las mismas produce un problema. Pues, aunque ninguna de estas legislaciones puede determinar o tener un influjo sobre la otra ${ }^{6}$, el suelo a donde se ejercen ambas legislaciones es, sin embargo, la experiencia. Esto es (Kant, 1991; AA, V, 174):

(...) el suelo sobre el cual se erige su dominio y es ejercida su legislación es siempre únicamente el compendio (Inbegriff) de los objetos de toda experiencia posible, en tanto que ellos no sean tomados más que como meros fenómenos.

Aun cuando ambas legislaciones son independientes «se restringen incesantemente en sus efectos en el mundo de los sentidos» (Kant, 1991; AA, V, 175). Así, aunque el dominio de la libertad se representa a su objeto, la voluntad, como cosa en sí misma, los efectos de ésta, esto es, las acciones de un agente libre, aparecen en el mundo de los sentidos,

\footnotetext{
$6 \quad$ Así, se afirma (Kant, 1991; AA, V, 195): «El concepto de la libertad no determina nada con respecto al conocimiento teórico de la naturaleza; nada, igualmente, el concepto de la naturaleza en vista de las leyes prácticas de la libertad, a tal punto que no es posible tender un puente de un dominio al otro».
} 
objeto del dominio de la naturaleza. De allí que surja inevitablemente el problema de pensar no sólo la ausencia de contradicción entre ambas legislaciones (cuestión tratada, como señalamos arriba, en la tercera antinomia de la CRP), sino además la posibilidad de la realización de la libertad en la experiencia. Esta cuestión es el problema del enlace entre la teoría y la práctica ${ }^{7}$. En los términos de Kant (1991; AA, V, 176):

Por mucho que se consolide un abismo inabarcable entre el dominio del concepto de la naturaleza, como lo sensible, y el dominio del concepto de la libertad, como lo suprasensible, de modo tal que no sea posible ningún tránsito desde el primero hacia el segundo (...) éste, sin embargo, debe tener sobre aquél un influjo, a saber, debe el concepto de la libertad hacer efectivo en el mundo de los sentidos el fin encomendado por su leyes.

\section{La facultad de juzgar como medio de enlace entre la teoría y la práctica}

Según su propia declaración de intenciones, mediante la CFJ Kant (1991; AA, V, 170) «concluy[e] con [su] negocio crítico». Con esto, es de presuponerse que la obra no sólo completa la búsqueda de principios trascendentales, sino que promete el hallazgo del tránsito entre la filosofía teórica y práctica, el cual todavía en la CRPr no había sido aún encontrado (según vimos arriba: Kant, 2007b; AA, V, 091).

Originariamente la CFJ había sido planeada como una «Crítica del gusto». Así, en una carta célebre a C. L. Reinhold fechada el 31 de diciembre de 1787 Kant (1902ss, AA, X, 513-5) afirma haber descubierto un principio a priori referido al sentimiento de placer y displacer, mediante el cual se añadía una parte al sistema de la filosofía (que hasta el momento se dividía en parte teórica y parte práctica). ${ }^{8}$ En el curso de la ela-

\footnotetext{
7 Allison (2001: 201/4) afirma que la solución de la tercera antinomia entre naturaleza y libertad en CRP se plantea «en el orden trascendental». En la CFJ el problema se plantearía en un nivel «empírico-antropológico», esto es, desde el "punto de vista práctico» del agente actuante en el mundo de los sentidos. Por eso, hacia el final de la Introducción definitiva Kant (1991; AA, V, 196) habría señalado que se ocupa del problema del tránsito en conexión directa con el «sujeto como ser sensible, o sea, como hombre».

8 La primera indicación que sugiere la redacción de una «Crítica del gusto» por parte de Kant se encuentra referida en una carta de J. Bering a Kant del 28 de mayo de 1787, de la cual se infiere que la obra estaba anunciada en el catálogo de la feria de Leipzig. Véase: Kant, 1902ss; AA, X, 487. Otras referencias epistolares a la redacción y, en general, la historia de la construcción del texto pueden consultarse en el ya citado texto de Tonelli (1954).
} 
boración de la obra, entre marzo de 1788 y mayo de $1789^{9}$, Kant amplió el objeto de estudio hacia una «Crítica de la facultad de juzgar». La CFJ apareció en las pascuas de 1790 y se compone de dos partes principales, una primera, dedicada a la «Crítica del gusto», seguida por una «Crítica de la facultad de juzgar teleológica».

El concepto de facultad de juzgar había sido introducido por Kant ya en la obra de 1781/1787. En este caso, es una facultad subordinada o subsidiaria $^{10}$, pues consiste en la capacidad de aplicar las reglas dadas a ella por el entendimiento. Así, a diferencia de éste, según CRP, la facultad de juzgar no tiene reglas propias (véase: Kant, 2007a; CRP, A133136/ B172-175; A646/ B674; Kant, 1991; AA, XX, 212), sino que es un «talento peculiar» o un «ingenio natural» que no puede ser enseñado sino sólo ejercitado. En la CFJ Kant reconsidera la función meramente suplementaria de dicha facultad al poner al descubierto un principio a priori que actuaría en la base del desempeño de la facultad de juzgar. En consonancia con la CRP, en la Introducción definitiva a la CFJ, Kant (1991; AA, V , 179) señala que «la facultad de juzgar, en general, es la facultad de pensar lo particular en cuanto contenido bajo lo universal», pero propone ahora una distinción novedosa entre facultad de juggar determinante y facultad de juggar reflexionante que anuncia el descubrimiento de una nueva facultad.

La facultad de juzgar determinante consiste en la actividad de subsumir un particular bajo un universal o un concepto que nos es dado a priori y es heredera de la noción de facultad de juzgar de la CRP, cuya función es, según hemos dicho, meramente subsidiaria del entendimiento. La facultad de juzgar reflexionante consiste, por el contrario, en la actividad judicativa que se realiza cuando es el particular (y no el universal) aquello que nos es dado. En este caso, la facultad de juzgar debe salir a la búsqueda del universal a fin de realizar la actividad de subsunción del particular. Dado un particular, entonces, una representación cualquiera de cuyo concepto no tenemos noticia, es función de la facultad de juzgar reflexionante encontrar o formar un concepto mediante la comparación del particular

La última vez que Kant refiere la obra como «Crítica del gusto» es en carta a Reinhold fechada el 7 de marzo de 1788 (véase: Kant 1902ss; AA, X, 532). Recién en carta al mismo corresponsal con fecha 12 de mayo de 1789 por primera vez la obra es referida como «Crítica de la facultad de juzgar (en la que la 'Crítica del gusto' es sólo una parte)» (Kant, 1902ss; AA, XI, 039).

10 «Si definimos el entendimiento en general como la facultad de las reglas, entonces la facultad de juzgar consiste en la capacidad de subsumir bajo reglas, es decir, de distinguir si algo cae o no bajo una regla dada (casus datae legis)» (Kant, 2007a; CRP, A132/B171). 
con otras representaciones. La comparación, junto a la abstracción y la comprensión, es primordial en la actividad reflexionante ${ }^{11}$.

En la CFJ Kant (1991; AA, V, 169) defiende que la facultad de juzgar reflexionante es una facultad legislativa a priori, esto es, que implica para su ejercicio la actuación de un principio trascendental. Se trata de un principio muy «peculiar», mediante el cual «no es conocida ninguna cosa», pues «no es una regla objetiva» como aquellas del entendimiento, sino que es «meramente subjetivo». A dicho principio el filósofo lo denomina conformidad a fin (Zweckmäßigkeit). La conformidad a fin es el principio mediante el cual la facultad de juzgar reflexionante realiza la actividad de subsunción de particulares bajo conceptos que no son dados a priori y se divide en tres funciones principales (Tonelli, 1957: 8): la función lógico-formal (en la actividad de búsqueda y subsunción de universales empíricos en el contexto de las ciencias empíricas), la función estético-formal (el enjuiciamiento estético en general: el gusto y el sentimiento de lo sublime) y, finalmente, la función objetivo-material (en el enjuiciamiento teleológico de los organismos).

Pues bien, Kant afirma hacia el final de la Introducción definitiva que el principio de la conformidad a fin, en general, es aquél que permite el enlace entre la teoría y la práctica. Expresamente afirma (Kant, 1991; AA, V, 196):

(...) la facultad de juzgar, suministra el concepto mediador entre los conceptos de la naturaleza y el concepto de libertad, que hace posible el tránsito de la legislación teórica pura a la práctica pura, de la conformidad a ley

11 En la base de la actividad de «reflexionar» (reflectieren, Überlegen) o también «discernir» (facultas diiudicandi, Beurtheilungsvermögen) se encuentra la comparación entre representaciones. Kant (1991; AA, XX, 211) define la actividad de «reflexionar» en la Primera Introducción como un «comparar (vergleichen) y mantener reunidas (zusammen halten) representaciones dadas, sea con otras, sea con su facultad de conocimiento, en relación a un posible concepto mediante ella». Según Baeumler (1923: 139, 203), Christian Wolff es quien habría introducido el concepto de «reflexión» (Überdenken) en el sentido que es usado aquí por Kant (véase: Wolff, 1730: \$233; 1732: \$\$234-36, 257, 258, 283). En la así llamada Lógica Jäsche Kant (2001; AA, IX, 094) distingue las actividades cognitivas de la comparación y de la reflexión (como también, éstas de la actividad de la abstracción) en el marco de la exposición de los pasos (u operaciones) cognitivos requeridos para la «formación de conceptos». Así, la comparación es el acto de «mantener reunidas» las representaciones en relación con la unidad de conciencia; reflexionar es el acto de «comprender» (begreifen) dichas representaciones en una conciencia; abstraer, finalmente, es «separar» aquellos aspectos de dichas representaciones que las diferencian. El producto de las tres operaciones conjuntas son los conceptos (Begriffe). Longuenesse (1998 [1993]: 112) ha mostrado que estas tres actividades se corresponden con el uso reflexionante de la facultad de juzgar. Reflexionar sería, entonces, la actividad misma de comparar, abstraer y reunir bajo concepto. 
según la primera, al fin final (Endzweck) según la segunda, en el concepto de una conformidad a fin de la naturaleza; pues por este medio es conocida la posibilidad de ese fin final, que es el único que puede llegar a ser efectivo en la naturaleza y con el acuerdo de sus leyes.

Así, a través del principio de la conformidad a fin que regula la actividad de la facultad de juzgar reflexionante es posible definir un «modo de pensar» (Denkungsart) la naturaleza, específicamente, la naturaleza del «sujeto como ser sensible, esto es, como hombre» (Kant, 1991; AA, V, 196), en la cual sea posible hacer efectivos los fines de la libertad o, como Kant aquí los denomina, el «fin final» (véase además: Kant, 1991; AA, V, 176). Se trata de desarrollar, a través de la facultad de juzgar, un «modo de pensar» la naturaleza humana distinto al modo como conocemos dicha naturaleza.

\section{Los dos modelos de enlace entre la teoría y la práctica}

Los intérpretes han atendido a estos pasajes y acuerdan en que la facultad de juzgar es la que nos provee de la mediación requerida entre la teoría y la práctica. Esta facultad es la que nos permite pensar la realización de los fines de la libertad en el suelo de la experiencia. Se trata de introducir un «modo de pensar» la naturaleza diferente al modo como la conocemos. En consonancia con esto, según vimos ya en la Introducción, han afirmado los críticos que es o bien a través del gusto (Mertens, 1975: 234; Rohlf, 2008: 344; Schrader, 1953/1954: 205, 218), o bien mediante la facultad de juzgar teleológica (Yovel, 1989: 31; Düsing, 1968: 103, 105, 216; Bartuschat, 1972: 245; Anderson-Gold, 1981: 3; Wood, 1999: 309; Allison, 2001: 208/218, 2009: 41) que Kant propone la instanciación del tránsito en cuestión. En el presente apartado no tomaremos partido por ninguna de estas líneas de interpretación contendientes $^{12}$, pues nos interesa ocuparnos de un problema que es aún más primitivo.

Según defendemos, en la Introducción definitiva, Kant propone dos modelos diferentes para pensar el modo general como la facultad de juzgar establece el enlace entre teoría y práctica. La descripción del primer modelo se encuentra hacia el final de la sección II y la descripción del

12 Allison (2001: 209-18) defiende que el tránsito es efectuado tanto por la facultad de juzgar estética como por la facultad de juzgar teleológica (nos remite aquí al parágrafo $₫ 83$ de $\mathrm{KU}$ ). Más específicamente, defiende la tesis de la complementación o colaboración mutua entre ambos tipos de enjuiciamientos en la efectuación del tránsito en cuestión. La perspectiva de H. Allison nos parece la más probable. En el trabajo a continuación dejamos deliberadamente este problema sin resolver. 
segundo modelo en el apartado IX y último del texto. Sobre estos nos ocupamos a continuación.

En cualquiera de los dos casos el concepto de lo suprasensible tiene un papel fundamental. Hay un pasaje final de la Introducción definitiva donde se deja constancia de la importancia de este concepto en la resolución del problema del tránsito en cuestión. Kant (1991; AA, V, 196) afirma:

El entendimiento, a través de la posibilidad de sus leyes a priori para la naturaleza, da prueba de que ésta es conocida por nosotros sólo como fenómeno, y da con ella, a un tiempo, indicios de una substrato suprasensible suyo, pero deja a éste completamente indeterminado. Por su principio a priori del enjuiciamiento de la naturaleza según leyes particulares posibles de ésta, la facultad de juzgar procura a su suprasensible (tanto en nosotros como fuera de nosotros), determinabilidad por medio de la facultad intelectual. Pero la razón le da a ése mismo la determinación por medio de su ley práctica a priori; y así la facultad de juzgar hace posible el tránsito del dominio del concepto de la naturaleza al del concepto de la libertad.

Arriba vimos que aunque el dominio de la libertad determina prácticamente el reino de lo suprasensible, no nos provee, como tampoco el dominio de la naturaleza, de un conocimiento teórico de su objeto como cosa en sí, esto es, ambos dejan teóricamente indeterminado el reino de suprasensible. Lo suprasensible, sin embargo, es el campo de la pura «determinabilidad», esto es, puede y debe ser pensado mediante múltiples conceptos e ideas. Más aún, en algún pasaje de esta Introducción Kant (1991; AA, V, 175) señala que se trata de «un campo que ciertamente debemos ocupar con ideas para provecho del uso teórico como asimismo práctico de la razón».

Pues bien, hacia el final del apartado II el filósofo presenta lo que, según nuestra hipótesis, es el primer modelo para pensar el tránsito entre naturaleza y libertad a partir de un pensamiento acerca de lo suprasensible. Kant (1991; AA, V, 176) señala lo siguiente:

Tiene que haber, entonces, un fundamento de la unidad de lo suprasensible que está en la base de la naturaleza con aquél que el concepto de la libertad contiene prácticamente, cuyo concepto [de ese fundamento], aunque no alcance ni teórica y prácticamente para un conocimiento suyo, y no tenga, por tanto, ningún dominio propio, haga posible, sin embargo, el tránsito desde el modo de pensar según los principios de uno al [modo de pensar] según los principios del otro. 
El modo de pensar la naturaleza que permite la realización de los fines de la libertad en el suelo de la experiencia se realizaría, según parece afirmar aquí Kant, a través del pensamiento de un «fundamento de unidad de lo suprasensible». Se trata de pensar un principio de unidad que reúna lo suprasensible «que está en la base de la naturaleza» y los suprasensible que está contenido prácticamente en el concepto de libertad. De esta manera el tránsito se realiza a través del pensamiento de un principio superior de lo suprasensible en tanto fundamento de los dominios de la naturaleza y de la libertad.

Ahora, en el apartado IX Kant parece ofrecernos otro modelo para pensar el enlace entre la teoría y la práctica. Retomando el problema que hemos desarrollado arriba el filósofo (Kant, 1991; AA, V, 196) afirma:

Pero si los fundamentos de determinación de la causalidad según el concepto de libertad (...) no son de constatar en la naturaleza y lo sensible no puede determinar lo suprasensible en el sujeto, esto sin embargo es posible a la inversa (no, por cierto, en vista del conocimiento de la naturaleza, más sí de las consecuencias que del primero se siguen para la segunda), y está contenido ya en el concepto de una causalidad por medio de libertad, cuyo efecto debe ocurrir en el mundo de acuerdo con éstas sus leyes formales, si bien la palabra causa, empleada a propósito de lo suprasensible, significa sólo el fundamento para determinar la causalidad de las cosas naturales de acuerdo con sus propias leyes naturales, con vista a un efecto, pero también al mismo tiempo acorde con el principio formal de las leyes racionales.

La realización de la libertad en el suelo de la experiencia, según el esquema que parece presentarnos aquí Kant, no nos refiere, como sí lo hacía antes, a un fundamento de unidad superior entre lo suprasensible subyacente a la naturaleza y lo suprasensible en tanto determinado por la libertad. Por el contrario, la realización parece aquí más directa. Kant señala que lo suprasensible por libertad en el sujeto puede o, incluso, debe (pues está «contenido en el concepto de una causalidad por medio de la libertad») determinar lo sensible en el sujeto. ¿Cómo se realiza dicha determinación? La causalidad por libertad contiene el «fundamento para determinar la causalidad de las cosas naturales». En sentido general, en los términos con los que venimos desarrollando esta cuestión, esto significa que el dominio de la libertad contiene el fundamento del dominio de la naturaleza, esto es, el fundamento de la causalidad según la ley natural. Ya no se trata de reunir bajo un primer principio dos legislaciones independientes, no se trata de explicar la posible concordancia entre naturaleza y libertad a partir de un único fundamento de origen. Lo que Kant parece sugerir aquí es que una de estas legislaciones, la legislación prácti- 
ca, se encuentra en el fundamento de la legislación teórica. Sería entonces pensable la realización de los fines de la libertad en el suelo de la experiencia porque en el fundamento del dominio de la naturaleza, en el fundamento de los conceptos a priori mediante los cuales conocemos los objetos en la intuición, subyacería la ley práctica. De esta manera, Kant parece presentarnos en el apartado IX de la Introducción definitiva un modelo alternativo al modo como se realizaría el tránsito entre naturaleza y libertad según el apartado II de este mismo texto.

Notemos, finalmente, que bajo estas dos soluciones al problema del enlace se conserva la independencia, que Kant no habría estado dispuesta a ceder, de las legislaciones en cuestión. Según el primer modelo, el pensamiento de la coincidencia entre dos legislaciones completamente separadas, se debe a una armonía pre-establecida supuesta como fundamento de unidad común de aquéllas; de acuerdo al segundo modelo, si bien el dominio de la libertad es puesto como fundamento del dominio de la naturaleza, esto no significa que pueda el primero intervenir sobre la legislación del segundo; por el contrario, es la serie entera de la ley natural aquella que es pensada bajo la ley por libertad. El dominio de los conceptos de naturaleza conserva, de esta manera, su independencia, al menos, relativa respecto al dominio práctico.

\section{REFERENCIAS}

-Allison, H. E. (2001). Kant's Theory of Taste. A reading of the Critique of Aesthetic Judgment. Cambridge: Cambridge University Press.

-Allison, H.E. (2009). Teleology and history in Kant. En A. Oksenberg-Rorty y J. Schmidt (ed.). Kant's Idea for the Universal History with a Cosmopolitan Aim. A Critical Guide. New York: Cambridge University Press.

-Anderson-Gold, Sh. (1981). Teleology and Radical Evil: An Interpretation of the Concept of Species Character in Kant's philosophy of History. Michigan: University Microfilms International.

-Baeumler, A. (1923). Kants Kritik der Urteilskraft. Ibre Geschichte und Systematik. Halle: Max Niemeyer Verlag.

-Bartuschat, W. (1972). Zum systemischen Ort von Kants Kritik der Urteilskraft. Frankfurt am Main: Vittorio Klostermann.

-Bauch, B. (1917). Immanuel Kant. Leipzig-Berlin: Göschen'sche Verlagshandlung.

-Baumgarten, A. G. (1757 [1739]). Metaphysica. Halle: Hemmerde.

-Cassirer, E. (1994 [1918]). Kants Leben und Lehre. Damstadt: Wiss. Buchges.

-Cassirer, H. W. (1970 [1938]). A commentary on Kant's Critique of Judgement. New York: Metheuen Library Reprints.

-Cohen, H. (1889). Kants Begründung der Aesthetik. Berlin: Dümmler.

-Driesch, H. (1924). Kant und das Ganze. Kantstudien, 29 (B II), 365-376. 
-Düsing, K. (1968). Die Teleologie in Kants Weltbegriff. Bonn: H. Bouvier u. Co. Verlag.

-Hartmann, M. (1926). Biologie und Philosophie. Berlin: Max Planck Institut.

-Horkheimer, M. (1925). Kants Kritik der Urteilskraft als Bindeglied zwischen theoretischer und praktischer Philosophie. Stuttgart: Verlag von W. Kohlhammer.

-Kant, I. (1902ss). Kants gesammelte Werke. Berlin: Preussische/Deutsche Akademie der Wissenschaften.

-Kant, I. (1991). Critica de la facultad de juggar. (Pablo Oyarzún Trad.). Caracas: Monteávila.

-Kant, I. (2001). Lógica. (Traducción de María Jesús Vázquez Lobeiras). Madrid: Akal.

-Kant, I. (2004). Sobre el uso de principios teleológicos en la filosofía. (Traducción de Nuria Sánchez). Logos. Anales del Seminario de Metafísica, 37, 7-47

-Kant, I. (2007a). Crítica de la razón pura. (Mario Caimi Trad.). Buenos Aires: Colihue.

-Kant, I. (2007b). Crítica de la raźón práctica. (Rovira Armengol Trad.). Buenos Aires: Lozada.

-Lerussi, N. (2014). Acerca de la analogía de la razón con lo orgánico. Reflexiones en torno a la expresión epigénesis de la razón pura en Kritik der reinen Vernunft B \$27. En M. Caimi (ed.), Estudios kantianos (págs. 113-136). Buenos Aires: Prometeo.

-Lerussi, N. (2013). La teoría kantiana de las razas y el origen de la epigénesis. Studia Kantiana. 15, 85-102.

-Longuenesse, B. (1998 [1993]). Kant and the Capacity to Judge. Sensibility and Discursivity in the Transcendental Analytic of the Critique of Pure Reason. New Jersey: Princeton University Press.

-McLaughlin, P. (1989). Kants Kritik der teleologischen Urteilskraft. Bonn: Bouvier Verlag.

-Mertens, H. (1975). Kommentar zur Ersten Einleitung in Kants Kritik der Urteilskraft. Zur systematischen Funktion der Kritik der Urteilskraft für das System der Vernunftkritik. München: Johannes Berchmans Verlag.

-Rohlf, M. (2008). The Transition from Nature to Freedom in Kant's Third Critique. Kantstudien, 99 (3), 338-360.

-Schrader, G. (1953/1954). The Status of Teleological Judgment in the Critical Philosophy. Kantstudien, 45, 204-235.

-Stadler, A. (1874), Kants Teleologie und ihrer Erkenntnisstheoretische Bedeutung. Berlin: Hanwitz \& Grossamnn.

-Tonelli, G. (1954). La formazioni del testo della Kritik der Urteilskraft. Reven International de Philosophie, 8, 423-448.

-Tonelli, G. (1957/8). Von den verschiedenen Bedeutungen des Wortes Zweckmässigkeit in der 'Kritik der Urteilskraft'. Kantstudien, 49, 154-166.

-Turro, S. (1996). Tránsito de la naturaleza a la historia en la filosofía de Kant. Ciudad de México: Anthropos.

-Ungerer, E. (1922). Die Teleologie Kants und ibre Bedeutung für die Logik der Biologie. Berlin: Verlag von Gebrüder Borntraeger. 
-Vaihinger, H. (1913 [1911]). Die Philosophie des als Ob: System der theoretischen, praktischen und religiösen Fiktionen der Menschheit auf Grund eines idealistischen Positivismus. Berlin: Reuther \& Reicher.

-Wolff, Ch. (1730). Philosophia prima sive ontologia. Frankfurt: Renger.

-Wolff, Ch. (1732). Psichologia empirica. Frankfurt: Renger.

-Wood, A. (1999). Kants Ethical Thought. Cambridge: Cambridge University Press.

-Yovel, Y. (1989). Kant and the Philosophy of History. New Jersey: Princeton University Press.

-Zammito, J. (1992). The Genesis of Kant's Critique of Judgment. Chicago \& London: The University of Chicago Press.

Sumario: Introducción; 1. Planteamiento del problema del enlace entre la filosofía teórica y práctica: dos legislaciones sobre el mismo suelo de la experiencia; 2. La facultad de juzgar como medio de enlace entre la teoría y la práctica; 3. Los dos modelos de enlace entre la teoría y la práctica; Referencias. 